\title{
EXPERIMENTAL AND THEORETICAL STUDY OF BINARY DROPLET HEAD-ON COLLISIONS IN MEMS
}

\author{
Ya-Pu Zhao, F. C. Wang, J. T. Feng \\ State Key Laboratory of Nonlinear Mechanics, \\ Institute of Mechanics, Chinese Academy of Sciences, \\ Beijing 100190, China \\ E-mail: yzhao@imech.ac.cn
}

\begin{abstract}
The experimental and theoretical investigations into the headon collision between a landing droplet with another one resting on the PDMS substrate were addressed in this talk. The colliding process of the two droplets was recorded with highspeed camera. Four different responses after collision were observed in our experiments: complete rebound, coalescence, partial rebound with conglutination, and coalescence accompanied by conglutination. The contact time between the two colliding droplets was found to be in the range of 10-20 milliseconds. For the complete bouncing case, Hertz contact model was applied to estimate the contact time of the binary head-on colliding droplets with both the droplets considered as elastic bodies. The estimated contact time was in good agreement with the experimental result.
\end{abstract}

Keywords: Droplet collision, Rebound, Coalescence, Conglutination, Contact time, Hertz contact theory

\section{Introduction.}

Considerable attention has been attracted for droplet collision dynamics over a very wide time span. In 1879, Lord Rayleigh noted that small droplets can bounce in collisions with a larger pool of water $[1,2]$. As natural phenomena and common knowledge, cloud droplet collision and coagulation are helpful for the formation of precipitation of rainfalls. Recently, raindrops have been used to harvest electric energy for microelectromechanical systems (MEMS) from a piezoelectric system [3]. Droplet impingement also plays an important role in many industrial applications, including ink-jet printing, spray painting and coating, rapid spray cooling of hot surfaces.
Polydimethylsiloxane (PDMS) elastomer has been widely used as a versatile material in the fabrication of microfluidic devices, rapid prototyping and nanolithography. The well-known advantages of PDMS include its optical transparency, chemical inertness and non-flammable property [4-6]. Motivated by such practical applications in its fundamental importance and industrial applications, the head-on impact of a droplet with another one resting on a horizontal PDMS substrate is studied both experimentally and theoretically in this talk.

\section{Experimental}

Two kinds of solid substrates were adopted in our experiments: PDMS membrane (Sylgard 184, Dow Corning, USA; ratio of the base to curing agent $=10: 1$ ) of $1.82 \mathrm{~mm}$ in thickness, and PDMS films fabricated directly on the indium-tin-oxide (ITO) glass, cleaned thoroughly by the sonicleaning method. PDMS films were spread onto the ITO glass with spin coating by spin coater (KW-4A). The relationship between the spin speed and thickness of the PDMS film was obtained in our previous research [7]. The thickness of the PDMS film was $7.6 \mu \mathrm{m}$, as measured with the Surface Profiler (Dektek II A).

In our experiment, the droplet was placed on the solid substrate and remained as a drop with a definite contact angle $\theta$ between the liquid and solid phases, which was measured with the OCA20 system (precision $\pm 0.1^{\circ}$ ) from Dataphysics, Germany, using a sessile drop method. Droplets were dropped to the substrate with a syringe needle of the system. When droplet 1 was deposited to the substrate, it kept vibrating for a while, before reaching a steady state, and then droplet 2 was dropped and collided with droplet 1 (Fig. 1). The entire impact process was recorded with high-speed photography of $400 \mathrm{FPS}$ 


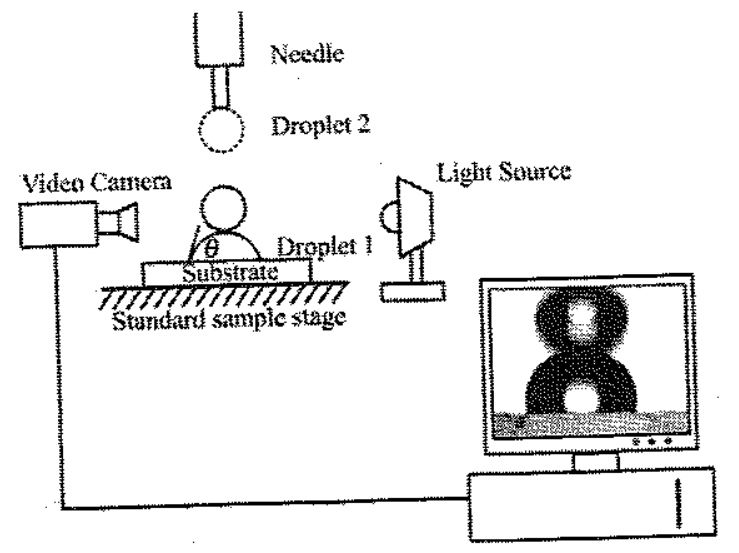

Figure 1. Experimental set-up.

(frame per second). Two kinds of droplets (pure water and soapy water with concentration of $2 \%$ ) were tested, under the temperature of $23.0^{\circ} \mathrm{C}$ and the relative humidity of $31.0 \%$.

\section{Results and discussion}

Four different types of response after collisions were observed: (i) Droplet 2 bounced completely like an elastic ball. (Fig. 2) In this case of complete rebounds, both droplets exhibited obvious elasticity. A simple model will be developed to estimate the contact time later.

(ii) The two droplets coalesced directly without rebound, permanently merging into one drop. (Fig. 3) Coalescences occurred much more frequently than complete rebounds.

(iii) Conglutination occurred when droplet 2 bounced and the two droplets separated successfully. (Fig. 4)

(iv) Droplet 2 rebounded and then coalesced together with droplet 1 due to the conglutination. (Fig. 5) Experiments also indicate that two droplets of pure water cannot separate successfully due to the conglutinations.

\section{Energetics and rebound time estimation}

The collinear impact of two elastic spheres is a solved problem according to Hertz's static theory of elastic contact [8]. When two liquid droplets collide together, the contact time $\tau$ is takes the form as [9]

$$
\tau=\frac{4 \sqrt{\pi} \Gamma(2 / 5)}{5 \Gamma(9 / 10)}\left(\frac{\xi^{2}}{k^{2} v}\right)^{1 / 5}
$$

where $\xi=m_{1} m_{2} /\left(m_{1}+m_{2}\right)$ is the reduced mass, $v$ is the relative velocity; $k=4 \sqrt{R_{1} R_{2} / R_{1}+R_{2}} / 5 E^{*}, R_{1}$ and $R_{2}$ are the curvature radii of the contact surfaces, $E^{*}=0.75\left[\left(1-\sigma_{1}^{2}\right) / E_{1}+\left(1-\sigma_{2}^{2}\right) / E_{2}\right], E_{1}, E_{2}$ and $\sigma_{1}, \sigma_{2}$ are the Young's moduli and the Poisson's ratios of the two droplets, respectively; $\Gamma(*)$ is the Gamma function. The subscripts 1

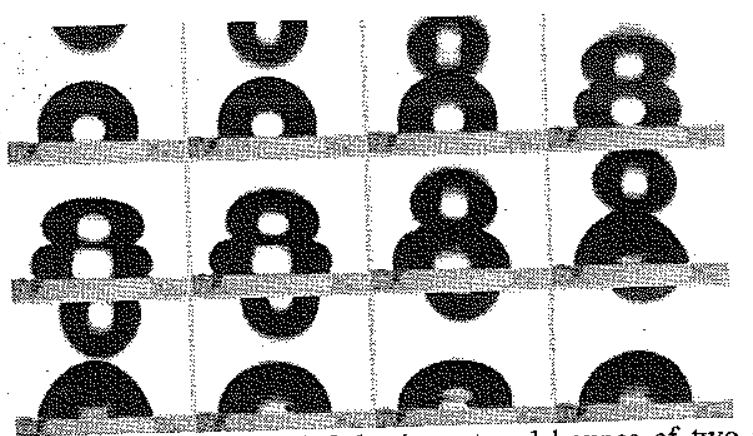

Figure 2. Sequential images of the impact and bounce of two pure water droplets. The solid substrate is PDMS film coated on ITO glass.

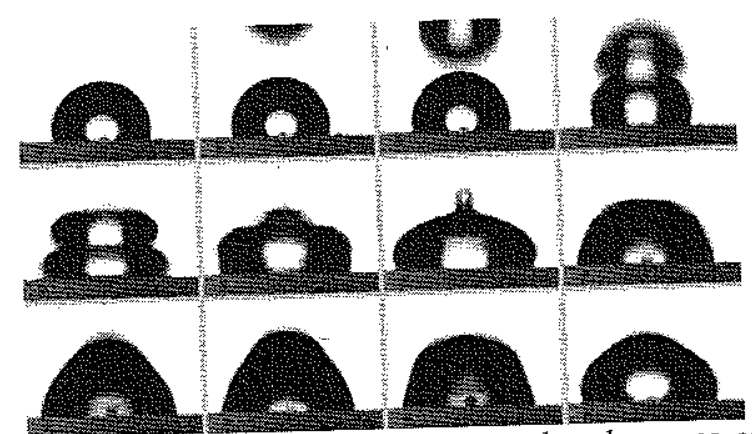

Figure 3. Sequential images of the impact and coalescences of two pure water droplets. The solid substrate is the same as that in Fig. 2.

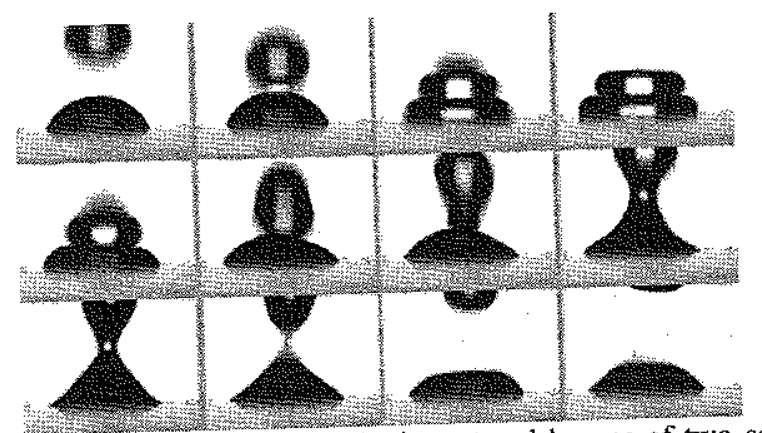

Figure 4 . Sequential images of the impact and bounce of two soapy water droplets. The solid substrate was PDMS membrane.

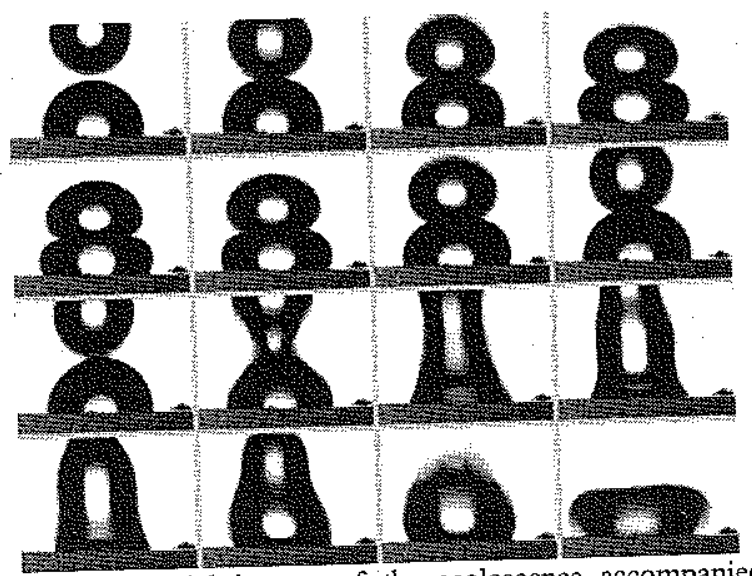

Figure 5. Sequential images of the coalescence accompanied by conglutination. The solid substrate is the same as that in Fig. 2. 
and 2 indicate quantities pertaining to droplet 1 and droplet 2 , respectively.

We take the Laplace pressure, $E=\gamma / R$ as its equivalent module [10]. The Poisson's ratio of both pure water and soapy water is taken as $\sigma_{1}=\sigma_{2}=0.5$ as in an incompressible condition.

In our model, droplet 1 is rest before collision, so the relative velocity is equal to the impact velocity of droplet 2 . The volume of the droplets can be measured experimentally (10.0 $\mu L$ for water and 5.0 $\mu L$ for soapy water). For these droplets, $R_{1}$ and $R_{2}$ will be known from the initial shape. Since the gravity force is neglected for the Bond number, $B o<1$, droplet 1 remains in the shape of an approximate spherical segment on the solid substrate and droplet 2 is in the shape of a sphere falling down.

The contact time measured in our experiments for pure water is about $17.5 \mathrm{~ms}$ and the theoretical result with our model as calculated by Eq. (1) is about $29.2 \mathrm{~ms}$. The experimental contact time for two soapy water droplets is about $15.0 \mathrm{~ms}$, while the theoretical solution is about $27.0 \mathrm{~ms}$. They are in quite good agreement.

It should be noted that ours is just a simple model with a number of factors neglected such as: (i) Part of the deformation energy stored in the solid substrate with respect to the overall deformation of the droplets during the collision. (ii) The elastic oscillations of the droplets during the collision.

\section{Conclusion}

In this paper, the head-on impact of the binary liquid droplets was studied experimentally and theoretically. Responses after the collision can be distinguished into four cases: complete rebound, coalescence, partial rebound after conglutination, and coalescence accompanied by conglutination. Hertz contact theory is introduced to estimate the contact time. The theoretical results agree very well with the experimental data. The authors believe that the droplet collision dynamics will remain an attractive scientific problem for a long time due to its various applications from aerospace to nanotechnology.

\section{Acknowledgments}

This work was jointly supported by the National Basic Research Program of China (973 Program, Grant No. 2007CB310500) and National Natural Science Foundation of China (NSFC, Grant No. 10772180).

\section{References}

[1] Rayleigh, L., 1945, "The Theory of Sound," Dover Publication, New York.

[2] Rayleigh, L.,1879, "On the capillary phenomena of jets" Proc. R. Soc. London, 29, pp. 71-97.

[3] Guigon, R., Chaillout, J.J., Jager, T., and Despesse, G., 2008, "Harvesting raindrop energy: experimental study," Smart Mater. Struct., 17, pp. 015039.
[4] Bodas, D., and Khan-Malek, C., 2006, "Formation of more stable hydrophilic surfaces of PDMS by plasma and chemical treatments," Microelectron. Eng., 83, pp. 12771279.

[5] Feng, J.T., and Zhao, Y.P., 2008, "Influence of different amount of $\mathrm{Au}$ on the wetting behavior of PDMS membrane," Biomed. Microdevices, 10, pp. 65-72.

[6] Feng, J.T., and Zhao, Y.P., 2008, "Experimental observation of electrical instability of droplets on dielectric layer," J. Phys. D: Appl. Phys., 41, pp. 052004

[7] Dai, W., and Zhao, Y.P., 2007, "The nonlinear phenomena of thin polydimethylsiloxane (PDMS) films in electrowetting," Int. J. Nonlinear Sci. Numer. Simul., $10(4)$, pp. 519-526.

[8] Hertz, H.,1881, "Über die Berührung fester elastischer Körper, "J. Reine Angew. Math., 92, pp. 156-171

[9] Landau, L.D., and Lifschitz, E.M., 1986, "Theory of Elasticity," Pergamon Press, Oxford.

[10] Richard, D., Clanet, C., and Quere, D., 2002, "Surface phenomena-Contact time of a bouncing drop," Nature, 417 , pp. 811 . 